\title{
High level amplification of N-MYC is not associated with adverse histology or outcome in primary retinoblastoma tumours
}

\author{
DM Lillington ${ }^{*,}$, LK Goff', JE Kingston², Z Onadim ${ }^{3,4}$, E Price ${ }^{3,4}$, P Domizio ${ }^{5}$ and BD Young' \\ 'Cancer Research UK, Department of Medical Oncology, St Bartholomew's and the Royal London NHS Trust, London, ECIM 6BQ, UK; ${ }^{2}$ Paediatric Oncology, \\ St Bartholomew's and the Royal London NHS Trust, London, ECIA 7BE, UK; ${ }^{3}$ Retinoblastoma Unit, St Bartholomew's and the Royal London NHS Trust, \\ London, ECIA 7BE, UK; ${ }^{4}$ Department of Pathology, St Bartholomew's and the Royal London NHS Trust, London, ECIA 7BE, UK; ${ }^{5}$ Department of \\ Histopathology, St Bartholomew's and the Royal London NHS Trust, London, ECIA 7BE, UK
}

Twenty-five primary retinoblastoma tumours were analysed by real-time quantitative polymerase chain reaction to determine the genomic copy number of the N-MYC gene (2p24) relative to the copy number for REL, B2M, ALB, AFIO and MLL. Twenty-one of these tumours were shown by Comparative Genomic Hybridization to contain variable copy number increases of chromosomal material mapping to 2p. High level amplification ( $>30$-fold) of N-MYC was found in three tumours, none of which showed adverse histological features and all patients are surviving at between 54 and 108 months post enucleation. Furthermore, the three tumours associated with metastasis and adverse patient outcome showed normal N-MYC copy number. Although high level amplification of N-MYC is an unfavourable prognostic indicator in neuroblastoma, these data show no evidence of a correlation between amplification of N-MYC and adverse outcome in retinoblastoma.

British Journal of Cancer (2002) 87, 779-782. doi:10.1038/sj.bjc.6600532 www.bjcancer.com

(c) 2002 Cancer Research UK

Keywords: N-MYC; retinoblastoma; real-time quantitative polymerase chain reaction (RQ-PCR); outcome

Retinoblastoma $(\mathrm{Rb})$ is an intraocular malignancy affecting young children with a frequency of one per 20000 live births (Knudson, 1971). Mutation of the RB1 tumour suppressor gene, located at chromosome 13 band $\mathrm{q} 14$, is essential to the pathogenesis of $\mathrm{Rb}$. In hereditary cases $(40-45 \%$ of cases) there is a germline mutation of RB1, thereby conferring predisposition, and the second allele is inactivated by somatic mutation. This 'two hit' mechanism was first hypothesised by Knudson (Knudson, 1976) and later substantiated following cloning of RB1 (Friend et al, 1986). In patients with germline mutations, bilateral $\mathrm{Rb}$ tumours usually develop and patients have an increased risk of secondary cancers, in particular osteosarcoma and soft tissue sarcoma (Draper et al, 1986; Roarty et al, 1988). In the sporadic unilateral Rb tumours, both RB1 mutations are required in the same somatic cell to initiate tumour formation. The risk factors for the development of sporadic $\mathrm{Rb}$ are not clear but it has been suggested that exposure to human papilloma viruses may play a role (Orjuela et al, 2000). A variety of chromosomal abnormalities have been reported in $\mathrm{Rb}$ tumours (Benedict et al, 1983; Squire et al, 1985; Mitelman, 2001), including frequent gains of $6 p$ and $1 q$, although the impact of these additional events on tumorigenesis and prognosis has not been thoroughly investigated. A correlation between gain of $6 \mathrm{p}$ and the most malignant behaviour in $\mathrm{Rb}$ has been proposed (Oliveros and Yunis, 1995) and a relationship between N-MYC amplification, higher proliferation index (PI), advanced tumour stage and poor clinical outcome was suggested (Kim et

*Correspondence: DM Lillington; CRUK Medical Oncology Unit, Science Building, St. Bartholomew's Hospital Medical College, Charterhouse Square, London ECIM 6BQ, UK; E-mail: D.Lillington@cancer.org.uk

Received 8 March 2002; revised 28 May 2002; accepted 25 June 2002 al, 1999). It is well recognised in haematological malignancies and solid tumours, that a number of recurrent chromosomal abnormalities have independent prognostic importance (Ferrando and Look, 2000; Misra et al, 2000; Mrozek et al, 2000; Taylor et al, 2000; Mrozek et al, 2001) and, in particular, amplification of N-MYC is known to be associated with adverse outcome in childhood neuroblastoma (George et al, 2001; Lastowska et al, 2001). Retinoblastoma, like neuroblastoma, is thought to be a tumour of neuroectodermal origin and it shares a very similar pattern of metastasis to neuroblastoma, metastasising predominantly to bone, bone marrow, nodes and central nervous system. N-MYC is also a target for amplification in childhood $\mathrm{Rb}$ but whether this represents a molecular marker for the assessment of prognosis in this tumour type is not known. Real-time quantitative polymerase chain reaction (RQ - PCR) using the TaqMan system provides a rapid and sensitive method for the analysis of gene dosage (Goff et al, 2000; Tajiri et al, 2001). There was considerable variation, by CGH, in the extent of gain for 2p23-25 in these Rb tumours and thus RQ-PCR was used to determine N-MYC copy number and this was correlated with tumour histology and outcome.

\section{MATERIALS AND METHODS}

\section{Tumour samples}

Tumour samples were obtained following enucleation and DNA extracted using Nucleon BACC kits (Amersham International plc, UK) according to the manufacturer's protocol. Twenty-one tumour DNA samples were identified by CGH to show gain of $2 \mathrm{p}$ material and these were selected for RT-PCR analysis. In addition, tumour DNA from a further four patients, including three $(056,035,048)$ from the poor histo-prognostic risk (PR) category two of whom died, was analysed although none had 
evidence for gain of $2 p$ by CGH. Seven tumours were derived from six bilaterally affected patients and two patients had a positive family history (one bilateral and one unilateral case). The histological risk, clinical details, $\mathrm{CGH}$ findings, treatment and outcome are summarised in Tables 1 and 2. CGH was performed using Vysis CGH kits (Vysis, Inc., France) according to the recommended protocol. The risk factors were assigned according to the criteria of Khelfaoui et al (1996) and the study was approved by the East London and The City Health Authority Research Ethics Committee.

\section{Real-time quantitative polymerase chain reaction} (RQ-PCR)

RQ-PCR analysis was performed using the ABI Prism 7700 sequence detector system (Taqman assay). Primers for N-MYC were chosen from intron 2 with the assistance of software Primer Express version 1.0 (ABI/Perkin Elmer, Foster City, CA, USA). The Accession Number for the sequence used was Y00664. The N-MYC primer sequences were $5^{\prime}-3^{\prime}$ forward GAAGATATGTTTTGATTTTCATGCTTG, used at $300 \mathrm{nmol} \mathrm{l}^{-1}$ and reverse TGT-

Table I Comparison of clinical details and CGH findings in Rb tumours

\begin{tabular}{|c|c|c|c|c|c|c|c|c|c|c|}
\hline $\begin{array}{l}\text { Number of Tumours } \\
(n)\end{array}$ & $\begin{array}{l}\text { Poor histo risk } \\
\text { (\%) }\end{array}$ & $\begin{array}{l}\text { Uni/Bil } \\
(\%)\end{array}$ & $\begin{array}{c}\text { Family history } \\
\text { (\%) }\end{array}$ & $\begin{array}{c}\text { Abn by CGH } \\
\text { (\%) }\end{array}$ & $\begin{array}{l}+2 p \\
(\%)\end{array}$ & $\begin{array}{c}A m p(2 p 2) \underset{(\%)}{[a m p N-M Y C]} \\
\text { (\%) }\end{array}$ & $\begin{array}{l}+1 q \\
(\%)\end{array}$ & $\begin{array}{l}+6 p \\
(\%)\end{array}$ & $\begin{array}{l}-16 q \\
(\%)\end{array}$ & $\begin{array}{l}-16 \\
(\%)\end{array}$ \\
\hline CGH study $(n=49)$ & 27 & $65 / 35$ & 8 & 96 & 31 & 12 & 57 & 69 & 27 & 12 \\
\hline RT $-P C R$ study $(n=25)$ & 32 & $75 / 25$ & 8 & 100 & $\begin{array}{r}60 \\
(47)\end{array}$ & $\begin{array}{r}24 \\
{[(100)]}\end{array}$ & 33 & 33 & 20 & 12 \\
\hline Mairal et al, $2000(n=24)$ & 33 & $79 / 21$ & 13 & 96 & 21 & $\begin{array}{r}17 \\
{[(100)]}\end{array}$ & 50 & 54 & 21 & 25 \\
\hline Herzog et al, 200। $(n=26)$ & ND & $100 / 0$ & 0 & 65 & 12 & 4 & 38 & 44 & 31 & 4 \\
\hline
\end{tabular}

Poor histo risk=tumours with adverse histo-prognostic risk factors. Uni/Bil=Unilateral or Bilateral. Abn=Abnormal by CGH (+=gain; $-=$ loss). Amp(2p2)=amplification by CGH; [ampN-MYC=amplification confirmed by PCR]. ND=Not documented.

Table $2 \mathrm{CGH}, \mathrm{RT}-\mathrm{PCR}$, treatment and outcome of $25 \mathrm{Rb}$ tumours

\begin{tabular}{|c|c|c|c|c|c|c|}
\hline \multirow[b]{2}{*}{ Tumour } & \multirow[b]{2}{*}{ Gains } & \multirow[b]{2}{*}{ Losses } & \multicolumn{3}{|c|}{ RQ-PCR (N-MYC) } & \multirow[b]{2}{*}{ Status } \\
\hline & & & Amp & copy no* & Treatment & \\
\hline \multicolumn{7}{|l|}{ PR group } \\
\hline 004 & $|q, 2 p 23 p t e r| 4 q \mid, 3 q 24$ & $18 p$ & $6 p$ & 6.7 & E & A \\
\hline 017 & $\begin{array}{l}\text { 2p23pter,9q34, | 2p | 2pter, } \\
\text { |7q2 I qter, | 8q2 | q23, I pp | 2pter }\end{array}$ & $7 p 21$ p22,8q24,Xp22.3,Xq28 & $6 p$ & 2.3 & $\mathrm{E}+\mathrm{Ct}$ & A \\
\hline 041 & 2q2 I pter, 13 q2 1 qter, 1 5q23qter & 6q26qter & Ip| |q32,6p & 2.3 & E & A \\
\hline 005 & $|q, 2 p| 2 p t e r$ & $5 q 32 q$ ter, $13 q 14 q 22$ & $6 p$ & $\mathrm{~N}$ & $E+C t$ & $\mathrm{D}$ \\
\hline 035 & Ip3|pter,7p2 Ipter, 17p | |qter,19 & $5,6 q 2$ | qter, I 3q I 3qter & |q| |q24,6p & $\mathrm{N}$ & E & D \\
\hline 048 & |3q3 | qter, |4,20p | 2pter & $2 q 36 q t e r,|3 q| 4 q 2 \mid$ & $\mid q, 6 p$ & $\mathrm{~N}$ & $\mathrm{E}+\mathrm{RT} \rightarrow \mathrm{Ct}$ & $\mathrm{D}$ \\
\hline \multicolumn{7}{|c|}{ NAR group } \\
\hline $001^{\circ}$ & $|q, 5 p, 13 q 3|$ qter & & $6 \mathrm{p}$ & $\mathrm{N}$ & E & A \\
\hline $008(R)^{a}$ & 2p23,6p21.3pter & & & $\mathrm{N}$ & E & A \\
\hline $012^{\mathrm{a}}$ & $\mathbf{2 p}, 6 \mathrm{p}, 18$ & & & $\mathrm{~N}$ & $E(R)$ & A \\
\hline 052 & 2p24pter & & & $\mathrm{N}$ & E & A \\
\hline 029 & $\begin{array}{l}\text { |q,2p23pter,6q | 3p23,7q2 | qter, } \\
\text { | 8q | 2pter, | 9q | 2pter,20q | | pter }\end{array}$ & 2q31 qter,3q,6q22qter, 16q & & 2.1 & $\mathrm{E}$ & $A$ \\
\hline $033^{\mathrm{a}}$ & | q,2p23pter, | 3q22qter, I 5q | 4qter & $16,17 p$ & & 2.0 & $\begin{array}{c}E(R) \\
\operatorname{LSRT}(\mathrm{L})\end{array}$ & $A$ \\
\hline 038 & $|q, 2 p 23 p t e r, 2 p| 5 p 2|, 6 p, X p| \mid$ & $14 q 32,16 q$ & & 3.5 & E & A \\
\hline 009 & & $|3 q| 2 q 3 \mid$ & $2 q 12 p t e r$ & $\mathrm{~N}$ & E & A \\
\hline 044 & $12 q 24$ & $2 q 35 q \operatorname{ter}, 16 q 13 q 21$ & $|q, 2 p| 2 p t e r$ & 2.3 & E & A \\
\hline 051 & $\begin{array}{l}\text { I q25qter,2,5p | 3pter,7p,7q3 | qter, } \\
\text { 9p23pter, I 2q | 4q2 I, I 2q24,20q }\end{array}$ & Ip32pter, I 2p, 16, 19,Y & $2 p$ & 2.5 & $\mathrm{E}$ & $A$ \\
\hline 026 & 7q34qter,9q34, 14, 17p |3, 17q25, 19,20p 1 2pter & 3p25pter, I 3q33qter, 16q & $2 p 21 p 25$ & 99.6 & E & A \\
\hline 050 & Iq, | 3q | 2qter & $2 q 24 q 31,3 q|| q 21,6 q 22,8 p, 14 q 32,20 q \mid 3$ & 2p I 2pter & 41.0 & *** $\mathrm{C} t+\mathrm{E}$ & A \\
\hline 054 & Iq25,7q22q34, 13q21 q32 & $6 q 14 q 21$ & $2 p 22 p 24$ & 38.1 & E & A \\
\hline
\end{tabular}

Gains (CGH ratio $>1.25<1.5)$ or amplifications (CGH ratio $>1.5$ ) of $2 \mathrm{p}$ or N-MYC (RQ-PCR copy no $\geqslant 2.0$ ) are highlighted in bold. *Average ratio of N-MYC to reference gene. $\mathrm{N}=$ normal copy number, ${ }^{\mathrm{a}}$ Bilateral case. ${ }^{\mathrm{b}} \mathrm{Positive}$ family history of $\mathrm{Rb}$. $\mathrm{Ct}=$ chemotherapy. LSRT=lens sparing radiotherapy. $\mathrm{E}=$ enucleation. *** $\mathrm{Patient}$ failed chemotherapy and proceeded to enucleation. $A=a$ live. $D=$ dead. 
AGCATCATGTGCGCATTC, used at $900 \mathrm{nmol}^{-1}$. The Taqman probe for N-MYC carried a $5^{\prime}$ FAM reporter and a TAMRA quencher (Perkin Elmer, Warrington, UK). N-MYC probe sequence was 5'-(FAM)-ACAATAATTTTCTACCCCAGCGTGGTAGTCAATG-(TAMRA)-3', used at $100 \mathrm{nmol} 1^{-1}$. PCR amplification was performed as previously described (Goff et al, 2000). Each assay included 'no template' control and standard curves using placental DNA diluted over five logs. To ascertain N-MYC copy number, the copy number of B2M (15q21-22), ALB (4q11-13), MLL (11q23), AF10 (10p12) and REL (2p12-13) (reference genes) was also investigated. For each tumour the ratio of N-MYC copy number/ reference gene copy number was determined and a value above two considered indicative of amplification (Bieche et al, 1998). N-MYC copy number was deduced using a minimum of three reference genes selected on the basis of the CGH results being normal for those regions. All standard curves had a correlation coefficient of at least 0.99 . The primer oligonucleotides were synthesized by the Imperial Cancer Research Fund, Oligonucleotide Synthesis Service.

\section{RESULTS AND DISCUSSION}

The RQ-PCR results are summarised in Table 2. Three tumours showed greater than 10 -fold increase in N-MYC copy number (026, 050 and 054) at 100-, 41- and 38-fold respectively. Figure 1a represents the amplification plot for N-MYC and B2M from tumour 026 and a typical standard curve (B2M) is shown in Figure $1 \mathrm{~b}$. Two of the three patients (026 and 054) were diagnosed at less than 12 months ( 7 and 3 months, respectively) whilst the third patient (050) presented at 67 months. Contrary to the findings by Mairal et al (2000) that tumours with amplification of N-MYC show mild differentiation, all three tumours studied here were poorly differentiated. All three patients are alive at 108 (026), 65 (050) and $54(054)$ months post enucleation and although the tumours were poorly differentiated, none of them demonstrated either of the recognised adverse histological features, namely deep choroidal or retrolaminar invasion of the optic nerve. The four tumours reported by Mairal et al (2000) with amplification of N-MYC also lacked adverse histoprognostic risk

A

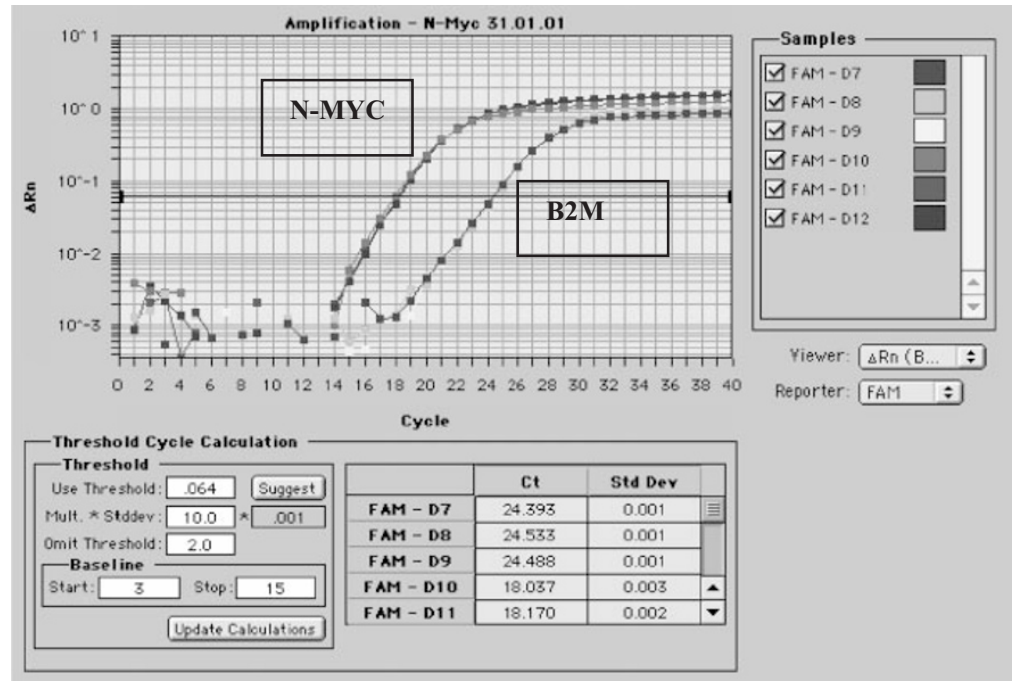

B

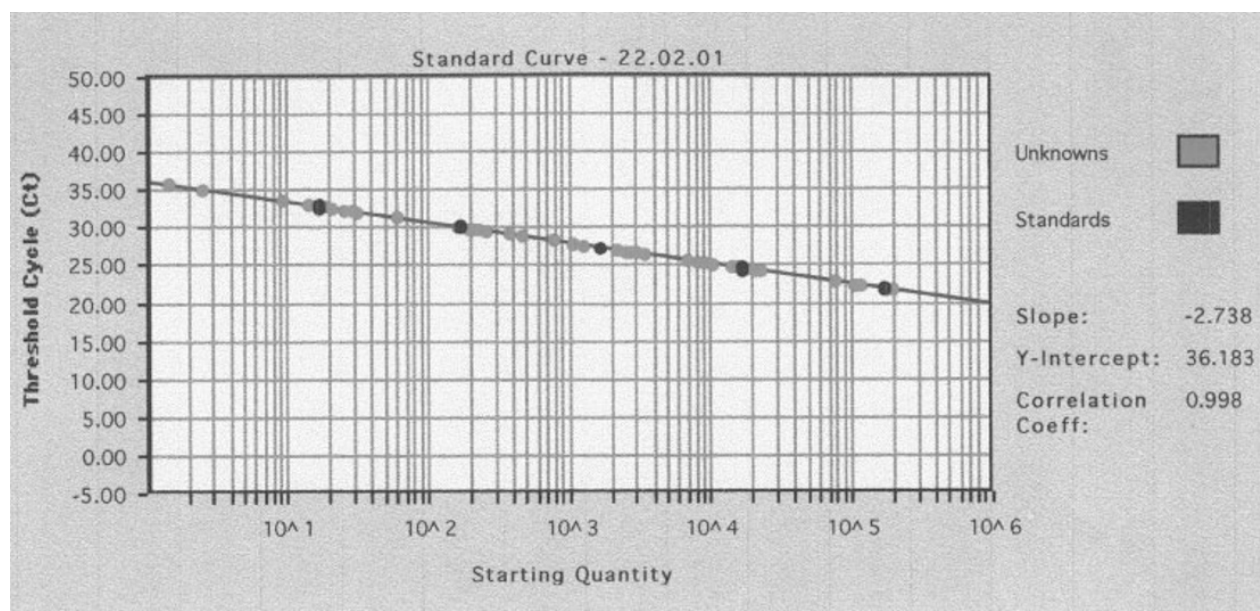

Figure I (A) RQ-PCR results showing the amplification plot for N-MYC and B2M (tumour 206). N-MYC:B2M copy number ratio=99.6. (B) Standard Curve for B2M. Black dots represent standard log dilutions, grey dots represent test DNA. The correlation coefficient for this standard curve was 0.998 . 
factors thereby strengthening a lack of association of multiple copies of N-MYC with poor outcome. Despite gain of $6 \mathrm{p}$ being a frequent $\mathrm{CGH}$ finding in $\mathrm{Rb}$ (34/49 tumours studied by $\mathrm{CGH}$, data not shown), all three tumours with high level amplification of N-MYC lacked gain of $6 \mathrm{p}$. It has been suggested that gain of $6 p$ is associated with more aggressive tumours (Oliveros and Yunis, 1995) and hence this lends further support to the hypothesis that high N-MYC copy number is not an adverse feature since these tumours also lacked gain of $6 \mathrm{p}$. The three patients who died following extra-ocular relapse (005, 035 and 048) showed no gain of N-MYC by RT - PCR and two also lacked gain of $2 p$ by CGH. Of those tumours with normal N-MYC copy number by RQ-PCR, there were five tumours showing gain of the entire $2 p$ arm by CGH and 4 showing gain of just the $2 p 2$ region. In all cases CGH suggested 0.5 -fold increase (i.e. one additional copy) and hence RQ-PCR may not be reliable enough to discern trisomy. The presence of any normal cells in the tumour will also hinder the ability to detect trisomy but this would have been applicable to the CGH experiments too. It is also possible that other genes mapping within $2 \mathrm{p} 2$ e.g. Id 2 may be the targets for low level gain in this region. Id2 belongs to a family of Id genes exerting an inhibitory effect on transcription by heterodimerising with basic helix-loop-helix proteins. Recently, Lasorella et al (2002) demonstrated the correlation of Id2 and $\mathrm{N}-\mathrm{MYC}$ expression and suggested that Id2 overexpression in

\section{REFERENCES}

Benedict WF, Banerjee A, Mark C, Murphree AL (1983) Nonrandom chromosomal changes in untreated retinoblastomas. Cancer Genet Cytogenet 10: $311-333$

Bieche I, Olivi M, Champeme M-H, Vidaud D, Lidereau R, Vidaud M (1998) Novel approach to quantitative polymerase chain reaction using real-time detection: application to the detection of gene amplification in breast cancer. Int J Cancer 78: $661-666$

Chen D, Gallie BL, Squire JA (2001) Minimal regions of chromosomal imbalance in retinoblastoma detected by comparative genomic hybridization. Cancer Genet Cytogenet 129: $57-63$

Draper GJ, Sanders BM, Kingston JE (1986) Second primary neoplasms in patients with retinoblastoma. Br J Cancer 53: 661-671

Ferrando AA, Look AT (2000) Clinical implications of recurring chromosomal and associated molecular abnormalities in acute lymphoblastic leukemia. Semin Hematol 37: $381-395$

Friend SH, Bernards R, Rogelj S, Weinberg RA, Rapaport JM, Albert DM, Dryja TP (1986) A human DNA segment with properties of the gene that predisposes to retinoblastoma and osteosarcoma. Nature 323: 643-646

George RK, Variend S, Cullinane C, Cotterill SJ, McGuckin AG, Ellershaw C, Lunec J, Pearson AD (2001) Relationship between histopathological features, MYCN amplification and prognosis: a UKCCSG study. United Kingdom Cancer Study Group. Med Pediatr Oncol 36: 169-176

Goff LK, Neat MJ, Crawley CR, Jones L, Jones E, Lister TA, Gupta RK (2000) The use of real-time quantitative polymerase chain reaction and comparative genomic hybridization to identify amplification of the REL gene in follicular lymphoma. Br J Haematol 111: 618-625

Herzog S, Lohmann DR, Buiting K, Schuler A, Horsthemke B, Rehder H, Rieder H (2001) Marked differences in unilateral retinoblastomas from young and older children studied by comparative genomic hybridization. Hum Genet 108: 98-104

Khelfaoui F, Validire P, Auperin A, Quintana E, Michon J, Pacquement H, Desjardins L, Asselain B, Schlienger P, Viehl P, Dufier J-L, Zucker J-M, Doz F (1996) Histopathologic risk factors in retinoblastoma. Cancer 77: $1206-1213$

Kim CJ, Chi JG, Choi HS, Shin HY, Ahn HS, Yoo YS, Chang KY (1999) Proliferation not apoptosis as a prognostic indicator in retinoblastoma. Virchows Archive 434: 301 - 305

Knudson AG (1971) Mutation and cancer: statistical study of retinoblastoma. Proc Natl Acad Sci USA 68: 820-823

Knudson AG (1976) Genetics and the aetiology of childhood cancer. Pediat Res 10: $513-517$ neuroblastoma may be a better prognostic indicator than $\mathrm{N}$ MYC amplification. Four tumours showing adverse histology did show gain of N-MYC; in tumours $017,08 \mathrm{~L}$ and 041 the increase was between 2-3-fold and in tumour 004 there was a 6.7 -fold increase.

In summary, molecular markers have allowed more precise assessment of an individual patient's prognosis in a number of malignant diseases and, in particular, N-MYC copy number is an important prognostic indicator in neuroblastoma and influences management. In the retinoblastomas studied in this series, tumours with amplification of N-MYC copy number ( $>30$-fold) were not associated with adverse histology or outcome, nor were they associated with gain of $6 \mathrm{p}$. Those tumours with adverse histology and adverse outcome (death following metastasis) did not exhibit either low or high copy number gain of N-MYC. The presence of multiple copies of N-MYC in Rb does not, therefore, appear to predict a poor clinical outcome.

\section{ACKNOWLEDGEMENTS}

The project was funded by the Joint Research Board, The Special Trustees of St. Bartholomew's Hospital, St. Bartholomew's Hospital, West Smithfield, London EC1A 7BE.

Lasorella A, Boldrini R, Dominici C, Donfrancesco A, Yokota Y, Inserra A, Iavarone A (2002) Id2 is critical for cellular proliferation and is the oncogenic effector of N-Myc in human neuroblastoma. Cancer Res 62: $301-306$

Lastowska M, Cullinane C, Variend S, Cotterill S, Bown N, O'Neill S, Mazzocco K, Roberts P, Nicholson J, Ellershaw C, Pearson AD, Jackson MS (2001) Comprehensive genetic and histopathologic study reveals three types of neuroblastoma tumors. J Clin Oncol 19: 3080-3090

Mairal A, Pinglier E, Gilbert E, Peter M, Validire M, Desjardins L, Doz F, Aurias A, Couturier J (2000) Detection of chromosome imbalances in retinoblastoma by parallel karyotype and CGH analyses. Genes, Chromosomes Cancer 28: $370-379$

Misra RR, Pinsky PF, Srivastava S (2000) Prognostic factors for hematologic cancers. Hematol Oncol Clin North Am 14: $907-924$

Mitelman F (2001) Mitelman database of chromosome aberrations in cancer, National Cancer Institute (http://cgap.nci.nih.gov/chromosomes/Mitelman).

Mrozek K, Heinonen K, Bloomfield CD (2000) Prognostic value of cytogenetic findings in adults with acute myeloid leukemia. Int J Hematol 72: $261-271$

Mrozek K, Heinonen K, Bloomfield CD (2001) Clinical importance of cytogenetics in acute myeloid leukaemia. Baillieres Best Pract Res Clin Haemato 14: $19-47$

Oliveros O, Yunis E (1995) Chromosome evolution in retinoblastoma. Cancer Genet Cytogenet 82: $155-160$

Orjuela M, Castaneda VP, Ridaura C, Lecona E, Leal C, Abramson DH, Orlow I, Gerald W, Cordon-Cardo C (2000) Presence of human papilloma virus in tumor tissue from children with retinoblastoma: an alternative mechanism for tumor development. Clin Cancer Research 6: 4010-4016

Roarty JD, McLean IW, Zimmerman LE (1988) Incidence of second neoplasms in patients with bilateral retinoblastoma. Ophthalmology 95: $1583-1587$

Squire J, Gallie BL, Phillips RA (1985) A detailed analysis of chromosomal changes in heritable and non-heritable retinoblastoma. Hum Genet 70: $291-301$

Tajiri T, Tanaka S, Shono K, Kinoshita Y, Fujii Y, Suita S, Ihara K, Hara T (2001) Quick quantitative analysis of gene dosages associated with prognosis in neuroblastoma. Cancer Lett 166: 89-94

Taylor CP, Bown NP, McGuckin AG, Lunec J, Malcolm AJ, Pearson AD, Sheer D (2000) Fluorescence in situ hybridization techniques for the rapid detection of genetic prognostic factors in neuroblastoma. United Kingdom Children's Cancer Study Group. Br J Cancer 83: 40-49 